\title{
Unmoderated Posters: Oncology - Kidney and Ureter
}

\begin{abstract}
UP-35
Percutaneous Radiofrequency Ablation of Renal Cell Carcinoma in Patients at High Risk for Surgery

Billia, Michele ${ }^{1}$; Mujoomdar, Amol' $^{2}$; Siddiqui, Khurram M. ${ }^{1}$; Mazzola, Clarisse R. ${ }^{1}$; Izawa, Jonathan ${ }^{1}$; Power, Nicholas ${ }^{1}$; Chin, Joseph ${ }^{1}$

${ }^{1}$ Departments of Urology, Western University; London Health Sciences Centre; London Victoria Hospital, London, ON, Canada; ${ }^{2}$ Departments of Radiology, Western University; London Health Sciences Centre; London Victoria Hospital, London, ON, Canada

Introduction and Objectives: Aim of this study was to assess safety and oncological outcomes of percutaneous radiofrequency ablation (PRFA) of renal cell carcinoma (RCC) in a prospective cohort of patients at high risk for surgery followed in a multidisciplinary environment.

Methods: 36 consecutive patients with a solitary kidney (7), and/or prior ipsilateral partial nephrectomy and/or ASA score $>3$ underwent PRFA under CT guidance. Indication for treatment was solitary renal mass $(<4 \mathrm{~cm}$ in size), multiple small masses and adrenal metastatic RCC in 29, 1 and 3 cases respectively. Prior to ablation, 3 percutaneous needle core biopsies were taken in all cases. Post-procedural CT scan was performed before discharge to exclude complications. All patients were followed with serial contrast-enhanced CT scans at 1,3,6 and 12 months. Endpoints of the study were safety, biopsy cancer detection rate, metastasis-free survival and OS. Results: We ablated 21 left-sided and 17 right-sided tumours, with mean size of $2.8 \pm 0.6 \mathrm{~cm}$. On the immediate postoperative CT scan an asymptomatic subcapscular hematoma was seen in 8 patients that did not require treatment. Technical failure was seen in 2 patients successfully treated with repeat RFA. There were 3 Clavien II complications: a pneumothorax at puncture and 2 urine leaks treated with antegrade stenting. In 8 cases $(24.2 \%)$, biopsy samples were insufficient for analysis, and Fuhrman grade could be scored in $8 / 27(29.6 \%)$ cases. We identified clear cell RCC in 36 and papillary RCC in 2 cases. With median follow-up of 18 (4-30) months, 2 patients had evidence of residual disease and successfully underwent a second RFA. There was no mortality and no pt had developed metastases at follow-up.
\end{abstract}

Conclusions: With meticulous patient selection, PRFA of RCC is a safe and effective treatment option for suboptimal candidates for renal surgery. Follow-up with serial CT scans is mandatory to identify persistent disease that can be treated with repeat PRFA.

\section{UP-36}

Utilization of Preoperative Investigations in Curative Renal Cell Carcinoma in a Population Based Cohort of Nova Scotia Patients Moideen, Nikitha'; Marzouk, Karim ${ }^{1}$; Thompson, Kara ${ }^{2}$; Wood, Lori ${ }^{3}$

${ }^{1}$ Dalhousie University, Halifax, NS, Canada; ${ }^{2}$ Research Methods Unit, Dalhousie University, Halifax, NS, Canada; ${ }^{3}$ Dalhousie University; QEII Health Sciences Centre, Halifax, NS, Canada

Introduction: Determining good quality care is essential in oncology. Obtaining appropriate preoperative risk-specific staging investigations for localized renal cell carcinoma (RCC) is a recognized quality indicator in RCC. The goal of this project was to determine the utilization and appropriateness of preoperative investigations in patients undergoing curative surgery for RCC.

Methods: The study is a population based retrospective review of all patients from mainland Nova Scotia (NS) having surgery for RCC from 2006-2010. Patients were identified through Cancer Care NS. REB approvals were obtained. Use of preop imaging and labwork within 3 months of surgery was recorded. "Appropriate" stage specific investigations were determined from National Comprehensive Cancer Network guidelines.

Results: 547 patients make up the study cohort. Median age was $62.6 \mathrm{yrs}$, $58 \%$ male, $37 \%$ had partial nephrectomy and $75 \%$ had clear cell pathology. $37 \%$ presented with local symptoms and $33 \%$ as an incidental finding. By 2002 pTNM criteria, $68 \%$ were stage I, $14 \%$ stage II, and $17 \%$ stage III. $78 \%$ had chest imaging (59\% had Xray and 69\% had CT). Of these, $60 \%$ of Xray and $69 \%$ of CT chest were ordered by urology and family docs respectively. For abdominal imaging, 51\% had ultrasound, 97\% had CT, and $14 \%$ had MRI. $98 \%$ had CBC, lytes, and creatinine, $18 \%$ had those plus $\mathrm{Ca} 2+$, and liver function tests (LFTs), and $16 \%$ had a urinalysis (UA). $88 \%$ of patients are alive and $11 \%$ have recurred. Stage specific utilization and outcomes in those without appropriate investigations will be presented. Conclusions: This study shows that chest imaging is underutilized in the entire cohort but CTs may be over-utilized in Stage I. Despite being recommended, tests such as LFTs, Ca2+ and UA are not done in the majority of patients. Overutilization and underutilization of healthcare resources is an obvious target for identifying good quality of care and targeting areas for improvement. This study provides the groundwork for this improvement.

\section{UP-37}

Lymphatic Drainage in Renal Cell Carcinoma: Back to the Basics Karmali, Riaz ${ }^{1}$; Suami, Hiroo ${ }^{1}$; Wood, Christopher G. ${ }^{2}$; Karam, Jose ${ }^{2}$ ${ }^{1}$ Department of Plastic Surgery, The University of Texas MD Anderson Cancer Center, Houston, TX, United States; ${ }^{2}$ Department of Urology, The University of Texas MD Anderson Cancer Center, Houston, TX, United States Introduction and Objectives: In renal cell carcinoma (RCC), lymphogenous metastasis is often detected outside of the expected hilar nodal region. A better understanding of lymphatic drainage in RCC would contribute to more accurate staging and an evidence-based consensus on retroperitoneal lymph node dissection.

Methods: We reviewed anatomic dissection studies since the 17 th century on lymphatic drainage of the kidney in humans. We summarized the results from recent sentinel lymph node (SLN) mapping studies in RCC patients. Results: Our review revealed anterior efferent lymphatic vessels from the right kidney drain to the paracaval, precaval, retrocaval, and interaortocaval LNs. Posterior efferent vessels drain to the paracaval, retrocaval, and interaortocaval LNs. From the left kidney, anterior vessels drain to the paraaortic and preaortic LNs while posterior vessels drain to the paraaortic and retroaortic LNs. Posterior vessels from both kidneys have also been observed to connect directly to the thoracic duct without passing through any retroperitoneal LNs. In 2 clinical studies $(n=24)$, drainage was localized to the paraaortic, paracaval, retrocaval, and interaortocaval LNs in 22 patients. Extraabdominal drainage was observed in 2 patients (mediastinal, pleural, int. mammary). Right-sided tumours $(n=16)$ drained primarily to paracaval, retrocaval and interaortocaval LNs. Left-sided tumours $(n=8)$ drained primarily to paraaortic LNs. Moreover, lymphovenous communication at the level of the renal vein exists in primates and rodents but remains unknown in humans.

Conclusions: Lymphatic drainage pathways described in past anatomic studies demonstrated a high similarity with the SLNs mapped in clinical studies. However, heterogeneity amongst SLN biopsy protocols may reduce the significance of results. Future anatomic studies should be carried out on pathological specimens with clinical studies using a standardized SLN biopsy approach. 


\section{UP-38}

Accuracy and Clinical Utility of Percutaneous Renal Core Biopsies and Fine Needle Aspirations

Patel, Premal'; Kaler, Kamaljot'; Kirkpatrick, Iain²; McGregor, Thomas ${ }^{1}$ ${ }^{1}$ Section of Urology, Department of Surgery, University of Manitoba, Winnipeg, MB, Canada; ${ }^{2}$ Division of Abdominal Imaging, University of Manitoba, Winnipeg, MB, Canada

Introduction and Objectives: The increasing incidence of renal cell carcinoma can largely be attributed to the detection of small renal masses on abdominal imaging. Often these masses are found incidentally and patients are asymptomatic. Previous studies have demonstrated the effectiveness and safety of percutaneous renal biopsies in the management of these patients, however use of biopsies has failed to gain widespread use. Our study aims to assess the accuracy of percutaneous biopsies and correlate this with clinical management and outcomes.

Methods: A retrospective chart review with ethics approval was undertaken for patients who had a percutaneous renal biopsy at the St. Boniface General Hospital from October 2012 to July 2013. Patient age, core biopsy and fine needle aspiration results, procedure, complications and if applicable, final surgical specimen pathology was extracted from patient charts.

Results: A total of 21 patients were found to have undergone percutaneous renal biopsies over the past year. Median patient age was 65.8 years old (range 50-80) with a male to female ratio of 4.25:1. Twenty-one patients had core biopsies and 19 patients had concurrent fine needle aspirations (FNA). Of the 21 core biopsies, 11 were found to be malignant, 6 were benign, 3 of which malignancy could not be excluded and 1 was nondiagnostic. Of the FNA, 12 were non-diagnostic, 4 were malignant and 3 were benign. Six patients underwent partial or radical nephrectomies during this period with core biopsy demonstrating final specimen pathology in 5 of the 6 patients and FNA demonstrating similar pathology in 2 of the 6 samples. All biopsies occurred without complications.

Conclusions: Our data to-date is consistent with literature in that percutaneous renal biopsies are safe as evident by lack of complications. Further our results show the limited utility of fine needle aspirations as they were largely non-diagnostic and add no additional information.

\section{UP-39}

The Canadian National Hereditary Kidney Cancer Needs Assessment Survey

Violette, Philippe ${ }^{1}$; Kamel-Reid, Suzanne ${ }^{2}$; Reaume, Neil'3; Jewett, Michael $^{4}$; Care, Melanie ${ }^{2}$; Basiuk, Joan ${ }^{5}$; Pautler, Stephen ${ }^{1}$

${ }^{1}$ Western University, London, ON, Canada; ${ }^{2}$ The University Health Network, Toronto, ON, Canada; ${ }^{3}$ The Ottawa Hospital Cancer Centre; University of Ottawa, Ottawa, ON, Canada; ${ }^{4}$ Princess Margaret Hospital, University Health Network, Toronto, ON, Canada; ${ }^{5}$ Kidney Cancer Research Network of Canada, Toronto, ON, Canada

Introduction and Objectives: Treatment of hereditary renal cell carcinoma (HRCC) requires a multidisciplinary approach that may involve medical oncologists, geneticists, genetic counselors, and urologists. The objective of our survey was to obtain current and accurate information about the use and perceived importance of genetic testing for HRCC from each of the above mentioned specialties.

Methods: A self-administered web-based survey was provided to medical oncologists, geneticists, genetic counselors, and urologists. The survey was created through an iterative process beginning with a focus group and repeated consultations with the members of the genetics division of the Kidney Cancer Research Network of Canada. The survey was designed to be exploratory and results were compared across provinces.

Results: The overall response was low, with 136 responders. Of the respondents, $42 \%, 33 \%, 19 \%, 5 \%$ were genetic counselors, urologists, medical oncologists and medical geneticists, respectively. The majority of respondents described their practice setting as primarily academic $62.7 \%$ vs. non-academic $37.3 \%$. Academic respondents tended to refer for genetic counseling more frequently than non-academic $67.2 \%$ vs. $48.6 \%, p<0.001$. The majority of respondents believed that genetic testing for HRCC were available $(82.8 \%)$, although $47.7 \%$ did not know which tests were available. This observation was consistent across provinces.
VHL testing was given highest priority among respondents. A lack of provider knowledge, clinical guidelines, institutional funding, access to genetic services and poor coordination between disciplines were cited as the main barriers to testing.

Conclusions: There is a significant need to increase provider knowledge of genetic testing for HRCC. These findings support the development of practice guidelines and national strategies to improve coordination of specialists and access to genetics services.

\section{UP-40}

Presentation, Pathology and Outcome of Non-clear Cell Renal Cell Carcinoma in a Population Based Cohort of Nova Scotia Patients

Himmelman, Jeffrey; Merrimen, Jennifer; Moideen, Nikitha; Marzouk, Karim; Thompson, Kara; Wood, Lori

Dalhousie University, Halifax, NS, Canada

Introduction and Objectives: Renal Cell Carcinoma (RCC) is the 8th most common cancer in Canada. The literature commonly cites that non-clear cell RCC (nccRCC) makes up about $20 \%$ of all RCC. NccRCC comprise a variety of subtypes with different genetic and clinical features as well as outcomes. The goal of this study was to characterize the subtypes, presentation, management and outcomes of nccRCC's of a population based cohort of patients from mainland Nova Scotia (NS).

Methods: A retrospective chart review was performed on patients with pathology proven nccRCC diagnosed between 2006-2010 on mainland NS. Patients were identified from Cancer Care NS. Appropriate research ethics board approvals were obtained. Descriptive statistics were used along with the method of Kaplan and Meier for time to event variables. Results: 697 patients were diagnosed with RCC during this time. 143 $(20.5 \%)$ unique patients had nccRCC with 148 kidney cancers. $71 \%$ were male, median age at diagnosis 61.5 years, and median mass size $4.85 \mathrm{~cm} .32 \%$ of patients presented with local symptoms and $16 \%$ were an incidental finding. Tissue diagnosis was made by radical nephrectomy $(50 \%)$, partial nephrectomy $(46 \%)$, biopsy $(3 \%)$ and autopsy $(1 \%)$. The most common pathology was papillary $(67 \%)$ followed by chromophobe $(20 \%)$ and other $(13 \%)$ which includes seven subtypes. $16 \%$ of patients had metastatic disease with $7 \%$ being diagnosed before surgery. Five-year survival of 116 patients presenting with localized disease was $93 \%(92.4 \%$ with papillary, $100 \%$ in chromophobe and $77.8 \%$ in other subtypes). In those presenting with metastatic disease the five-year survival was $27.3 \%$. Conclusions: This study describes a modern day population based cohort of patients with nccRCC. The incidence is similar to the published literature and is comprised of a number of different pathological subtypes with variable outcomes.

\section{UP-41}

Survival Outcomes for Squamous Cell Carcinoma in Patients Undergoing Renal Surgery: A Canadian Population Health Assessment

Lawson, Keith; Chan, Tony; Timilshina, Narhari; Alibhai, Shabbir; Bhatt, Jaimin; Finelli, Antonio

Department of Surgical Oncology, Princess Margaret Hospital, University of Toronto, Toronto, ON, Canada

Introduction and Objectives: Squamous cell carcinoma (SCC) of the renal pelvis is reported to account for less than $1 \%$ of upper urinary tract malignancies and portend abysmal survival rates relative to urothelial carcinomas. However, there is a paucity of literature supporting these figures with no contributing Canadian data.

Methods: We conducted a population level, retrospective, observational study utilizing administrative data from the Ontario Cancer Registry to identify patients diagnosed with SCC following renal surgery between 1995 and 2008. Histology codes were used to identify SCC patients and Kaplan-Meier analysis was utilized to determine SCC-specific mortality. Results: 26 cases of SCC were observed in the study period. The mean age of patients was 69 with a near $1: 1$ ratio of male to females. $81 \%$ of tumours were pT3 stage of higher with $19 \%$ presenting with lymph node metastasis. Primary mode of therapy included radical nephrectomy (75\%), nephroureterectomy (19\%) and ureterectomy (6\%). A $25 \%$ positive 
margin rate was observed. One patient received systemic chemotherapy. SCC-specific survival at 3 years was $23 \%$.

Conclusions: This study represents the first report on survival after renal surgery for SCC in Canada. Consistent with previous studies we observed that renal SCC is exceptionally rare and associated with a poor prognosis with patients often presenting late and with locally advanced disease. Ongoing tissue banking and research is required to improve outcomes associated with this deadly and understudied disease.

\section{UP-42}

Robotic Partial Nephrectomy for pT1b-T2 Tumours in 98 Patients Metcalfe, Charles ${ }^{1}$; Satkunasivam, Raj'; Yip, Wesley²; Abreu, Andre²; Aron, Monish'; Gill, Inderbir²; Desai, Mihir ${ }^{2}$

'University of Southern California Institute of Urology, Santa Monica, CA, United States; ${ }^{2}$ University of Southern California Institute of Urology, Los Angeles, CA, United States

Introduction and Objectives: Although robotic partial nephrectomy (RPN) is increasing in application, data on RPN for pT1b-T2 tumours are scarce. We report RPN in 98 patients with pT1b-T2 tumours, the largest singleinstitutional experience to date.

Methods: From our prospective database, 307 consecutive patients undergoing RPN (2009-2013) for pT1a tumours $<4 \mathrm{~cm}$ ( $\mathrm{n}=209$; group I) and pT1b-T2 tumours $>4 \mathrm{~cm}$ ( $\mathrm{n}=98$; group II) were identified. Patient demographics, tumour characteristics, perioperative and postoperative outcomes were retrospectively analyzed. pT2 tumours were present in 8 patients.

Results: See Table 1

Conclusions: Robotic partial nephrectomy is a safe and feasible option for highly selected patients with pT1b-T2 tumours $>4 \mathrm{~cm}$. Clinical and functional outcomes are comparable to those $<4 \mathrm{~cm}$. Longer follow-up is necessary to confirm oncologic outcomes.

Table 1. UP-42. Median (range) data or N (\%) data are presented

\begin{tabular}{|c|c|c|c|}
\hline & Group I & Group II & $p$ value \\
\hline No. patients & 209 & 98 & - \\
\hline Tumour diameter on $\mathrm{CT}(\mathrm{cm})$ & $2.8(1-4)$ & $5.3(4.1-14)$ & $<0.001$ \\
\hline R.E.N.A.L score & $7(4-11)$ & $9(5-11)$ & $<0.001$ \\
\hline Hilar tumours/solitary kidney & $13 \% / 7 \%$ & $21 \% / 8 \%$ & $\mathrm{n} / \mathrm{a}$ \\
\hline Operative time (hours) & 3.7 & 4.4 & $<0.0001$ \\
\hline Blood loss (mL) & 150 & 203 & $<0.0001$ \\
\hline Ischemia time (minutes) & 12 & 16 & 0.5 \\
\hline Zero-ischemia technique & $73(35 \%)$ & $35(36 \%)$ & 0.9 \\
\hline $\begin{array}{l}\text { Conversion to radical } \\
\text { nephrectomy }\end{array}$ & 1 & 5 & 0.014 \\
\hline Positive margins & 1 & 2 & $\mathrm{n} / \mathrm{a}$ \\
\hline Upgrade to $\mathrm{pT} 3$ & 9 & 8 & 0.19 \\
\hline Transfusion rate & $18(8 \%)$ & $18(18 \%)$ & 0.02 \\
\hline Hospital stay (days) & 3 & 4 & 0.016 \\
\hline $\begin{array}{l}\text { Intra/Post-op urologic } \\
\text { complications }\end{array}$ & $1 / 6$ & $0 / 7$ & $\mathrm{n} / \mathrm{a}$ \\
\hline Tumour recurrence/mortality & $1 / 0$ & $0 / 1$ & $\mathrm{n} / \mathrm{a}$ \\
\hline $\begin{array}{l}\% \text { change in discharge } \\
\text { creatinine }\end{array}$ & $0 \%$ & $19 \%$ & 0.002 \\
\hline $\begin{array}{l}\% \text { change in } 1 \text { month } \\
\text { creatinine }\end{array}$ & $7 \%$ & $18 \%$ & 0.0009 \\
\hline$\%$ change in discharge eGFR & $0.0 \%$ & $-18 \%$ & 0.002 \\
\hline$\%$ change in 1 month eGFR & $-12.1 \%$ & $-17.2 \%$ & 0.016 \\
\hline Mean follow-up (months) & $4.6(1-35)$ & $4.6(1-35)$ & $\mathrm{n} / \mathrm{a}$ \\
\hline
\end{tabular}

\section{UP-43}

Predicting Incidental Pathologic Up-staging of Clinical T1 Renal Masses

Metcalfe, Charles ${ }^{1}$; Santomauro, Michael $^{2}$; Chopra, Sameer ${ }^{2}$; Aron, Monish ${ }^{2}$; Gill, Inderbir²; Desai, Mihir ${ }^{2}$

${ }^{1}$ University of Southern California Institute of Urology, Santa Monica, CA, United States; ${ }^{2}$ University of Southern California Institute of Urology, Los Angeles, CA, United States

Introduction and Objectives: Clinical prediction of peri-nephric and hilar fat invasion (pT3) may be difficult for many small renal masses undergoing partial nephrectomy ( $\mathrm{PN}$ ). We report our experience with pathologic upstaging of cT1 renal tumours undergoing PN and attempt to identify possible clinical predictors.

Methods: Retrospective analysis of 236 renal tumours (159 cT1a, 77 T1b) treated with robotic PN was performed from a prospectively maintained database (2010-2013). Patient demographics, radiographic tumour characteristics, histopathologic data and oncologic follow-up were assessed. The likelihood of upstaging to pT3a was compared between cT1a versus cT1b tumours. Additionally, upstaged tumours were compared to those that remained pT1 for various clinical variables.

Results: Overall incidence of pT3a disease was $7 \%(17 / 236)$, including $6 \%$ for cT1a $(9 / 159)$ vs. $10 \%$ for cT1b $(8 / 77)(p=0.19)$. There were no differences between upgraded pT3a with to regards tumour size, RENAL score and centrality in both the cT1a or cT1b sub-groups. All pT3a tumours had negative resection margins and 15/17 had involvement of peri-nephric fat whereas 2/17 invaded renal sinus fat. The pT3a subgroup had similar distribution of Furhman grade when matched for clinical stage compared to tumours that remained pT1. Lymphovascular invasion and sarcomatoid histology was similar to those not upstaged. Pre- and 1 month postoperative estimated glomerular filtration rate was similar between pT3a vs. pT1 patients. Over a mean follow-up of 5 months (1-35), no patient with pT3a disease and 1 patient with pT1a disease had tumour recurrence $(\mathrm{p}=\mathrm{NS})$.

Conclusions: Incidental upstaging to pT3a disease remains difficult to predict from clinical or radiographic parameters and occurs with equal frequency in cT1a vs. cT1b tumours. While short-term follow-up show favorable outcome after partial nephrectomy, longer-term follow-up in a larger cohort of patients is required to confirm oncologic efficacy. 